\title{
Experimental Heat Transfer Study in an Intermediate Turbine Duct
}

\author{
Borja M. Rojo Perez ${ }^{*}$, Valery Chernoray ${ }^{\dagger}$, Martin Johansson ${ }^{\ddagger}$ and Maxim Golubev ${ }^{\S}$ \\ Chalmers University of Technology, SE-41296 Gothenburg, Sweden.
}

Due to demands from industry on improved efficiency, reduced $\mathrm{CO}_{2}$ and $\mathrm{NO}_{x}$ and decreased noise levels, the trend of future aero engines show that turbofan engines are designed with higher by-pass ratio. Two-spool and three-spool turbofan engines are designed with an intermediate turbine duct that connects the high-pressure turbine to the lowpressure turbine in the two-spool engine and two intermediate turbine ducts from HPT to intermediate pressure turbine (IPT) and IPT to LPT in the three-spool engines. The design of agressive intermediate turbine ducts (high radial offset for a short axial length) for these engines enables the possibility to increase the energy efficiency of the aero engine.

The flow and heat transfer in a turbine duct is very complex. The flow has large secondary structures and is sensitive to flow separation, which is difficult to predict with numerical methods. Limited information is available in open literature that can be used for validation of numerical methods.

This paper presents an experimental study of the heat transfer in an aggressive intermediate turbine duct. The aim of this study is to measure the of a surface temperature distribution and convection heat transfer coefficient with very high resolution and precision on a loaded guide vane which is located inside the intermediate turbine duct. Furthermore, the experimental results are compared to CFD carried out with ANSYS CFX.

This experiment was carried out in a state-of-the-art large-scale low-speed turbine facility at Chalmers University of Technology. The duct configuration investigated represents a modern counter rotating turbine design, with a flow turning structural vane. The facility includes a turbine stage which provides realistic inlet conditions into the duct and operates at realistic flow Reynolds number based on the ITD vane chord length. The measurements were performed by using an infrared camera.

The results shows that the heat transfer coefficient predicted in the computations close to the shroud is not well predicted. There can also be seen areas where there is flow transition and boundary layer transition.

\section{Nomenclature}

$C F D \quad$ Computational fluid dynamics

$H T C, h \quad$ Heat transfer coefficient, $\mathrm{W} / \mathrm{m}^{2} \mathrm{~K}$

HPT High pressure turbine

LPT Low pressure turbine

IPT Intermediate pressure turbine

ITD Intermediate turbine duct

$q^{\prime \prime} \quad$ Heat flux, $\mathrm{W} / \mathrm{m}^{2}$

$T \quad$ Temperature, $\mathrm{K}$

$\epsilon \quad$ Emissivity

$\sigma \quad$ Stefan-Boltzmann constant, W $/ m^{2} K^{4}$

$k \quad$ Thermal conductivity, $\mathrm{W} / \mathrm{mK}$

\footnotetext{
*PhD Student, Department of Applied Mechanics, Chalmers University of Technology

$\dagger$ Associate Professor, Department of Applied Mechanics, Chalmers University of Technology

${ }^{\ddagger}$ PhD Student, Department of Applied Mechanics, Chalmers University of Technology

$\S$ Post-doctoral fellow, Department of Applied Mechanics, Chalmers University of Technology
} 
$t \quad$ Silicone thickness, $\mathrm{m}$

Subscript

cd Conduction

cv Convection

$r \quad$ Radiation

wall Vane surface in contact with air

core Vane aluminum core

\section{Introduction}

In an aero engine the ITD is equiped with a thick structural vane that supplies, e.g, oil and wiring to the core of the engine from outside. Depending on configuration, these vanes can be either aerodynamically loaded or unloaded. The ITD is positioned directly downstream of the HPT, where the flow is very complex to predict due to its unsteadiness, secundary flows and high turbulence levels. Therefore, tip leackeage flow passing above the HPT unshrouded rotor blades, wakes and vortices are also important to be considered at the inlet of the ITD. Furthermore, the large area ratio between the inlet and outlet of the ITD can result in separation of the flow. One way of improving the efficiency of an aero engine is by increasing the area ratio or radial offset make the ITD shorter, i.e decreasing the weight of the ITD. In that case it becomes more difficult to predict the flow along the ITD and increases the risk of flow separation. However, this would further increase the risk of flow separation and hence also increase the difficulty in numerically predict the flow through this component.

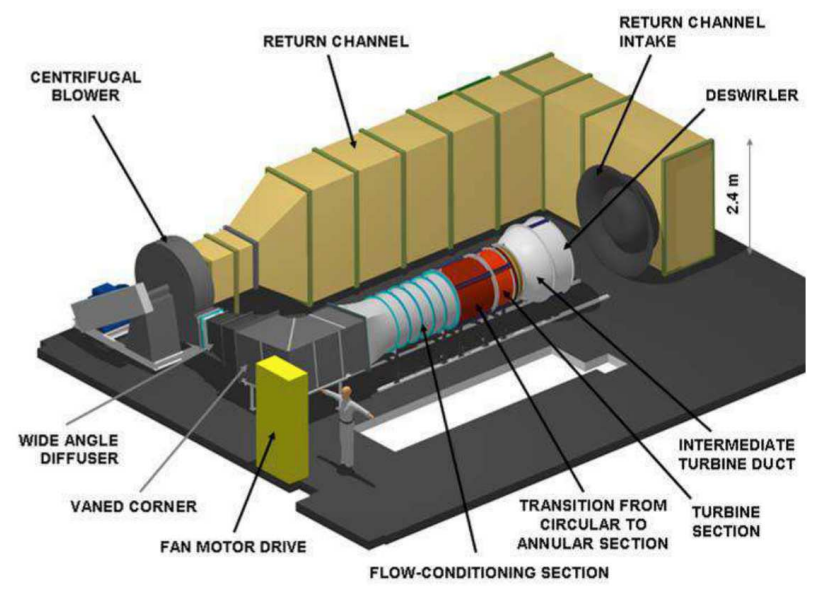

Figure 1. Experimental facility. ${ }^{1}$

In addition, the complexity of the flowfield makes the heat transfer prediction inside an ITD more difficult. Furthermore, the lack of experimental data in open literature related to the heat transfer in ITDs makes hard to design an optimized duct so that it can be calculated which material is the most suitable to stand heat loads and stresses at high temperatures without overdimensioning the duct.

For this study an experimental facility at Chalmers University of Technology was used which is specifically designed for investigations of the flow and heat transfer inside an ITD. It is a large-scale low-speed closed loop facility powered by a centrifugal blower of $110 \mathrm{~kW}$. This facility provides the possibility of studying the ITD flow with engine representative non-dimensional parameters due to the presence of the inlet HPT stage. The presence of an HPT stage upstream of the ITD ensures that realistic conditions in terms of flow angles, velocity and pressure distribution. Figure 1 shows a schematic of the facility. A more detailed description of the facility, although with a different ITD, can be found in previous studies ${ }^{1}$ and ${ }^{3}$.

The aerodynamic design of the current test section (see Fig. 2) was carried out by GKN Aerospace (previously Volvo Aero Corporation) and hardware implementation and flow field measurements was performed by Chalmers. ${ }^{2}$ In the present work the working section was instrumented for heat transfer measurements.

The measured data sets the base for a well-defined test case for CFD validation. The HTC measured is compared with CFD results in this paper. 


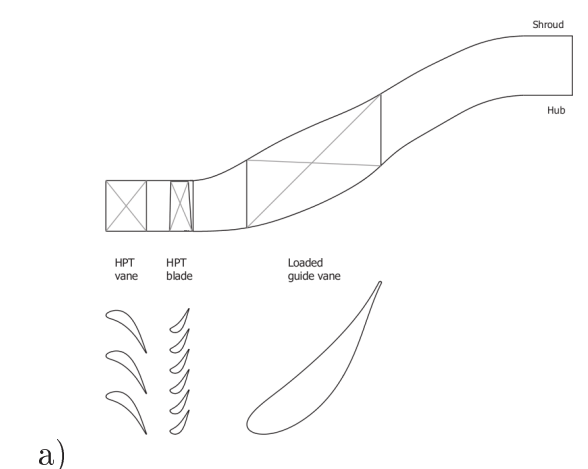

b)

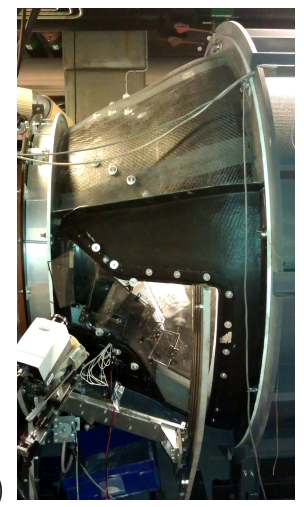

Figure 2. a) Schematic of the working section. b) Experimental setup.

\section{Methodology}

\section{II.A. Experimental Vane}

The composite vane for heat transfer measurements consists of a core with high thermal conductivity in order to create a well-defined boundary condition on the inside of the silicone insulating layer. Hence, there is a uniform temperature distribution in the volume. The insulating shell creates a large gradients of temperature (see Fig. 3). The core is made of aluminum and the shell of silicone of which has been glued with a constant thickness of $5 \mathrm{~mm}$ to the aluminium core (the thickness of glue is aproximately $2 \%$ of the silicone thickness). The vane is heated by 8 electrical cylindrical heaters placed within the aluminum core. These heaters are expanded during the experiment so that there is almost no contact resistance with the aluminum core and all the heat can be uniformly distributed along the holes length.

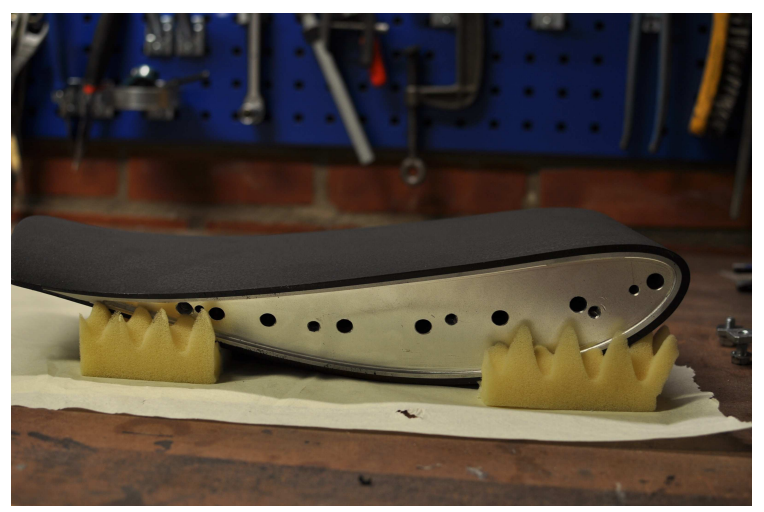

Figure 3. Test vane.

In order to validate the temperature uniformity, a CFD simulation has been performed where it was shown that for an average surface heat transfer coefficient of $100 \mathrm{~W} / \mathrm{m}^{2} \mathrm{~K}$, the maximum temperature difference in the aluminum core is $1^{\circ} \mathrm{C}$. This maximum temperature difference is mainly located close to the trailing edge, due to the small thickness of the aluminum core in that area and the larger distance to the closest heater.

The vane surface is covered with a black coating (Nextel Velvet Coating 811-21 from Mankiewicz Gebr. \& Co.) which has a high emissivity of 0.973 and low reflectivity in the IR range up to an angle of $60^{\circ}$. Furthermore, 130 markers are placed on the vane surface, which helps to map the measured temperature field onto physical coordinates, and also since it is not possible to take a complete picture of the pressure side or suction side at once with the IR-camera. Hence, during the post-processing of the images, the dots are used to overlap all the pictures obtained from the experiment. 


\section{II.B. Theoretical Model}

As a simplified model, a one-dimensional model is used as a first aproximation, while a comercial software is used when further post-procesing the measured data, in order to study the 3D heat conduction between the aluminium core and the silicone outer surface. The heat supplied to the insulating silicone shell from the core, via conduction, is transferred to the surroundings via convection and radiation (see Fig. 4). It has been estimated before the experiment was performed that the radiation heat flux would be around 5-10\% of the total heat flux. $T_{\text {wall }}$ is the temperature is measured by the IR camera. Knowing this value and the temperature of the aluminum core, the heat flux can be obtained on the surface as illustrated by the following equations.

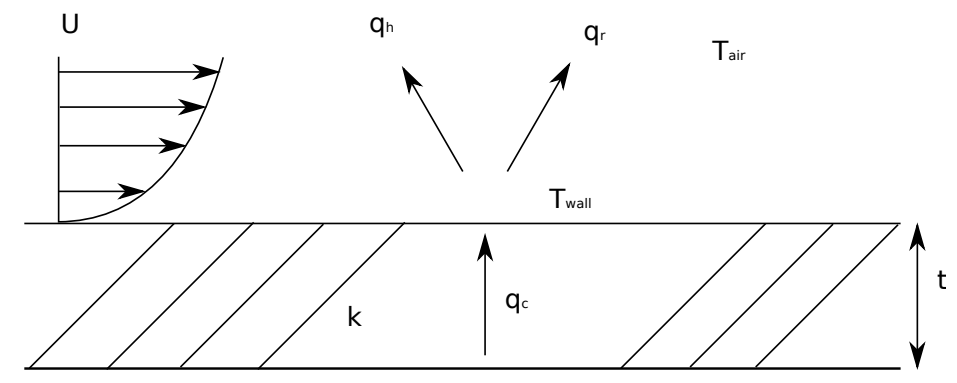

$T_{\text {core }}$

Figure 4. Simplification model for heat transfer measurement.

$$
\begin{aligned}
q_{c d}^{\prime \prime}=-k \frac{\partial T}{\partial x}=\frac{k}{t}\left(T_{\text {core }}-T_{\text {wall }}\right) & \text { Conduction heat flux } \\
q_{c v}^{\prime \prime}=h \Delta T=h\left(T_{\text {wall }}-T_{\text {air }}\right) & \text { Convection heat flux } \\
q_{r}^{\prime \prime} \approx \sigma\left(\varepsilon_{\text {wall }} T_{\text {wall }}^{4}-T_{e q}^{4}\right) & \text { Radiation heat flux }
\end{aligned}
$$

The equivalent temperature of the environment $T_{e q}$ is obtained empirically. This temperature takes into account reflections inside the ITD from the heated guide vane and the fact that the endwalls and guide vanes around the composite vane are almost at the freestream temperature during the experiment. If radiation is not taken into account, the error in peak temperature would be 15 and $6 \%$ respectively, only due to this. Applying the energy conservation equation as illustrated in Fig. 6 the following equation is obtained.

$$
q_{c}^{\prime \prime}=q_{c v}^{\prime \prime}+q_{r}^{\prime \prime}
$$

Then, HTC can be calculated solving the equation 4 .

$$
H T C=\frac{\frac{k}{t}\left(T_{\text {core }}-T_{\text {wall }}\right)}{T_{\text {wall }}-T_{\text {air }}}-\frac{\sigma\left(\varepsilon_{\text {wall }} T_{\text {wall }}^{4}-T_{e q}^{4}\right)}{T_{\text {wall }}-T_{\text {air }}}
$$

\section{II.C. Procedure}

Hub and shroud opening windows were designed in order to provide optical access for the IR-camera (see Fig. 2). Otherwise, the camera would not be able to measure the vane surface temperature due the high reflectivity of the window material at the measured IR wavelength interval, which in case of this camera it is $3-5 \mu \mathrm{m}$.

The vane surface temperature is measured with an IR camera (MWIR Phoenix Camera System). The camera was calibrated for the experimental conditions using an external source of temperature. In order to perform this calibration, an isolated aluminum plate which temperature was controlled by water that circulated through it controlled by a PI system.

During the measurements the aluminum core was kept at constant temperature of $65^{\circ} \mathrm{C}$ and the mean temperature of the freestream inside the duct far from the loaded vane surface is $28.5^{\circ} \mathrm{C}$. The uniformity of the core temperature was repeated controlled during the experiments using two thermoresistors (Pt 1000) 
Error on average HTC as a function of equivalent temperature

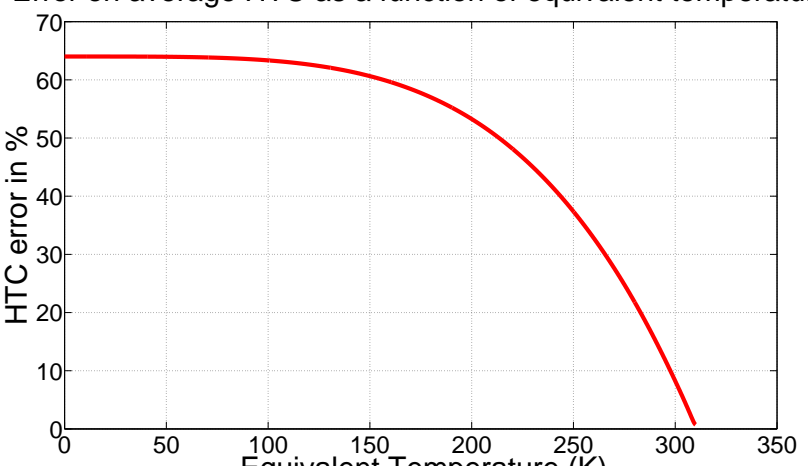

b)

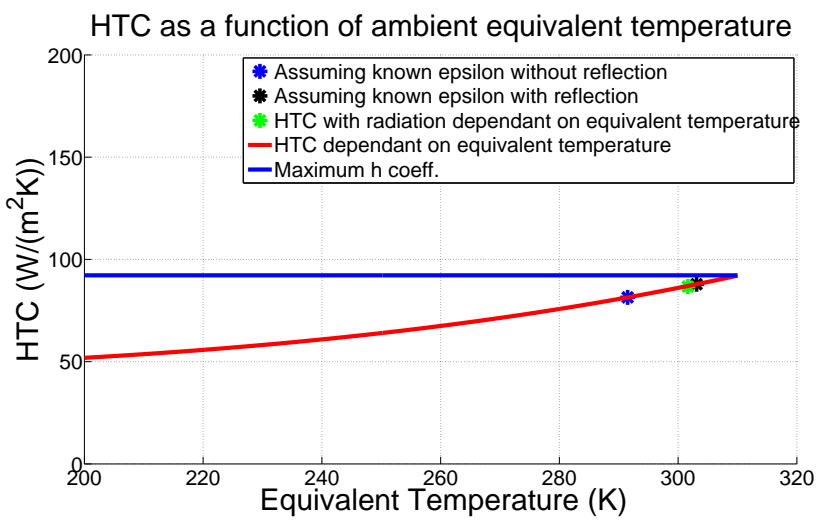

Figure 5. Error in HTC calculation due radiation flux.

situated inside the aluminum core during the experiment. The air temperature inside the ITD was measured with a thermoresistor (Pt100) and checked with a mercury thermometer.

During the experiment, 31 thermal images are taken with the IR-camera ( 17 for the pressure side and 14 for the suction side). The surface temperature from the images is mapped into physical coordinates, filtered and assembled into a single data file. The final surface temperature field is used as a Dirichlet boundary condition for a 3D heat flux calculations, together with the uniform core temperature, to obtaining the HTC.

The commercial solver FLUENT v.14 was used to perform the calculations. Then, the heat flux calculated is post-processed in Matlab, in order to add thermal radiation effects and study errors in the calculation of the HTC. Figure 5 shows how the error HTC calculation is dependent on the radiation heat flux (or equivalent temperature of the surrondings). From Fig. 5 it can be seen that all assumptions and measurements concerning the calculation of the equivalent temperature point that its value is around $300 \mathrm{~K}$ and that the error on HTC calculation without including radiation is around $7 \%$.

\section{Results}

\section{III.A. Experimental results}

Experimentally obtained surface temperature distribution along the vane is shown in Fig. 6, and the heat transfer coefficient map without the radiation correction is shown in Fig. 7. The contours of the heat transfer coefficient are typical for this component.

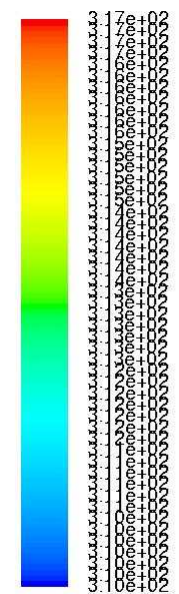

a)

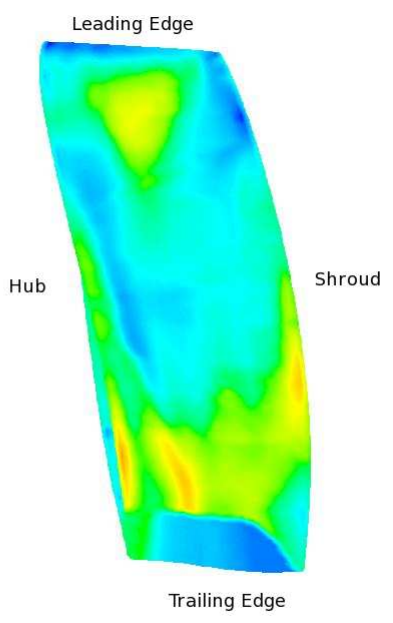

Trailing Edge

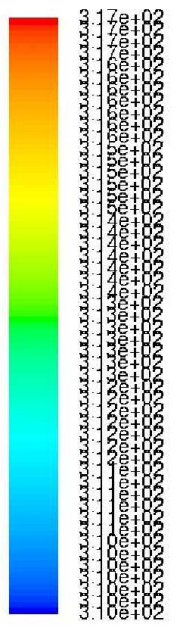

b)

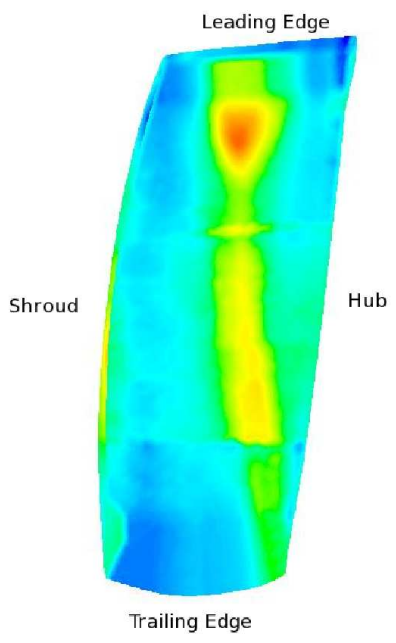

Trailing Edge

Figure 6. Experimentally measured vane surface temperature visualized in FLUENT. 
It is to be noted that the data close to the leading edge, on both the pressure and the suction side, has been extrapolated by FLUENT and cannot therefore be considered to be corrected. The same holds for the trailing edge. There are no measured data in these areas due to the problem of gaining optical access in the current setup.

The discrepancies seen on the pressure sides seen as sharp steps in the contours between hub and shroud, are due to a small step between the silicone patches.

The results clearly show the influence of the secondary flow and the tip leakage flow on the heat transfer on the pressure side of the vane. The tip leackage flow enters the duct at an angle very different from main flow's. This gives that instead of being aligned with the vane leading edge, the tip leackage flow is impinging on the pressure side, close to the shroud, and then rolls up into a vortex. This vortex is migrating towards the hub while travelling through the duct due to an adverse pressure gradient. The presence of vortex results in areas of increased heat transfer due to the higher velocity induced next to the wall, and higher local shear stress and increased mixing with the freestream flow.

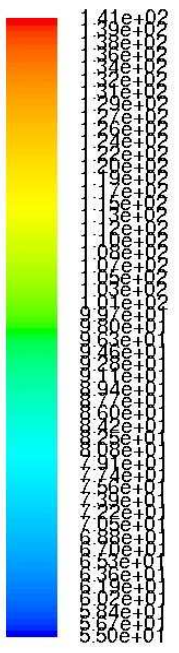

a)

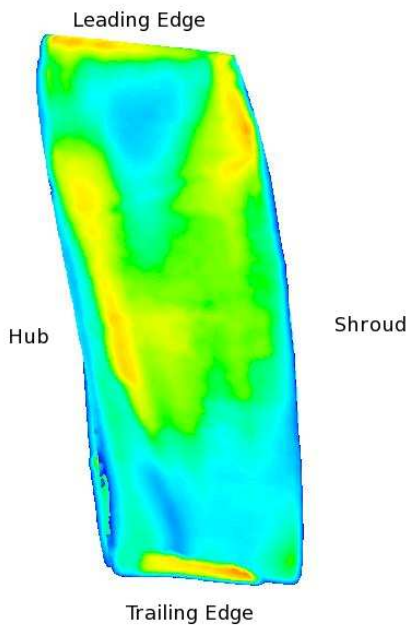

Trailing Edge

b)
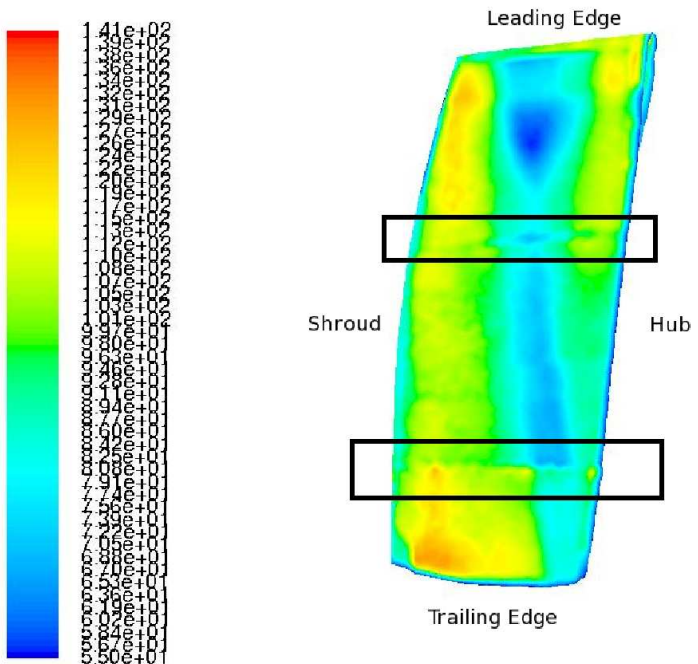

Figure 7. Calculated convection heat transfer coefficient map from experimental temperature distribution.

\section{III.B. CFD results}

In order to perform CFD heat transfer simulations, realistic boundary conditions are needed. In a previous study $\left({ }^{2}\right)$, inlet swirl angles, velocities and pressures were mapped at the inlet of the duct and are here used as circumferentially averaged boundary conditions at the domain inlet. The analysis was performed using ANSYS-CFX (v13.0), with a $k-\omega$ SST turbulence model. A second analysis was also performed using the Gamma Theta model to predict boundary layer transition. The mesh is of order 2.2 million cells and with resolved boundary layers. The results from these simulations can be seen in Fig. 8 and Fig. 9. 


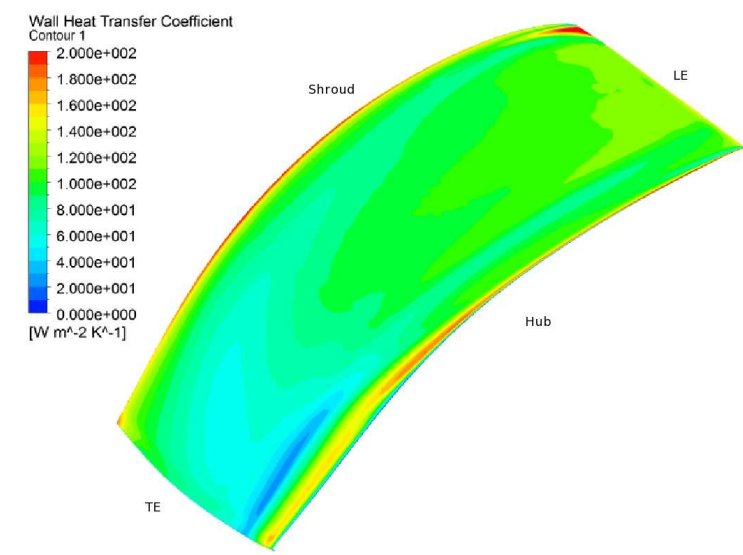

a)

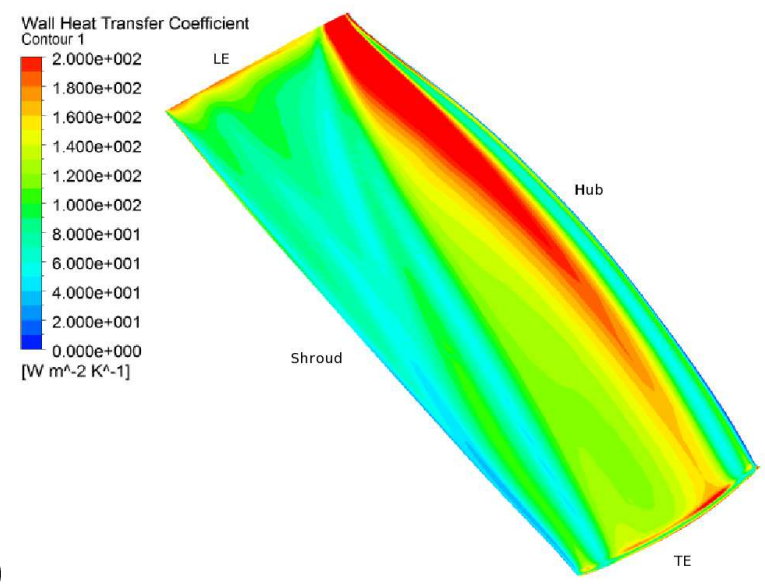

Figure 8. Calculated convection heat transfer coefficient map from CFX with $k-\omega$ SST.

Again, the influence of the tip leackage is clearly seen in the results of both analysis as an area of increased HTC close to the shroud on the pressure side. The HTC in this area is more than twice that of the rest of the vane. The effect of the tip leackage flow can also be seen on the suction side close to the leading edge as an area of increased HTC. Furthermore, the maximum values of HTC are seen on the leading edge. From this stagnation point, the boundary layers develop and grow when travelling downstream along the vane surface.

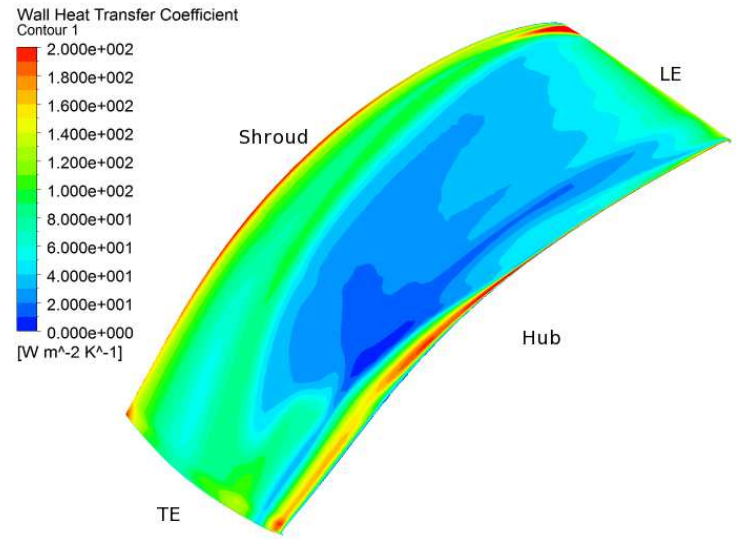

a)

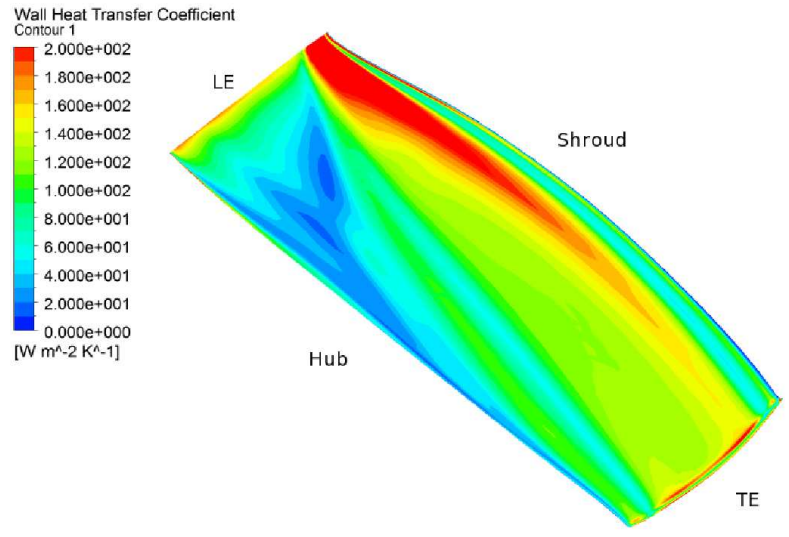

b)

Figure 9. Calculated convection heat transfer coefficient map from CFX with $k-\omega$ SST and Gamma Theta transition model.

\section{III.C. Compared results}

Figure 10 show numerical and experimental results at 10\%, 25\%, 50\%, 75\%, 90\% radial span.

It can be seen in Fig. 10 that the experimental data does not match with the predicted CFD results. The main difference is in the region where the tip leackage influences the flow, on the pressure side at $75 \%$ and $90 \%$. At $90 \%$ span the predicted HTC is more than two times the experimental one close to the leading edge. This gives that the tip leakage flow is not as strong as CFD predicts and therefore becomes as important as the vortices and secondary flow structures originating from the HPT stage.

In addition, when looking at the predicted result, using the Gamma Theta transition model, along the suction side at $10 \%, 25 \%, 50 \%$ and $75 \%$ it is evident that transition from laminar to turbulent boundary layer is present. Comparing with the experimental results, it is seen that the transition is significantly delayed in the predictions. For the remaining parts of Fig. 10 the transition is predicted closer to the leading edge.

Finally, excluding the pressure side at $75 \%$ and $90 \%$ as previously discussed, the predicted HTC without 
the transition model is of similar magnitude as the experimental data, although not capturing all the details.

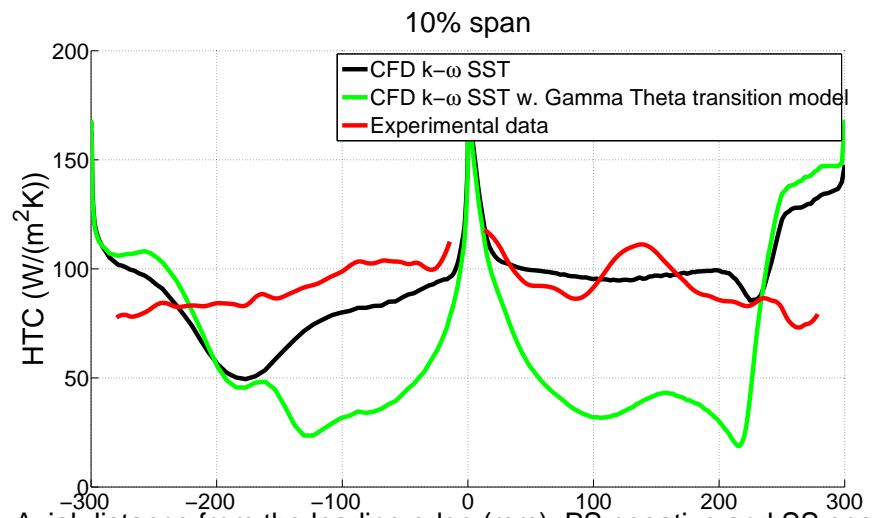

Axial distance from the leading edge $(\mathrm{mm})$. PS negative and SS positive

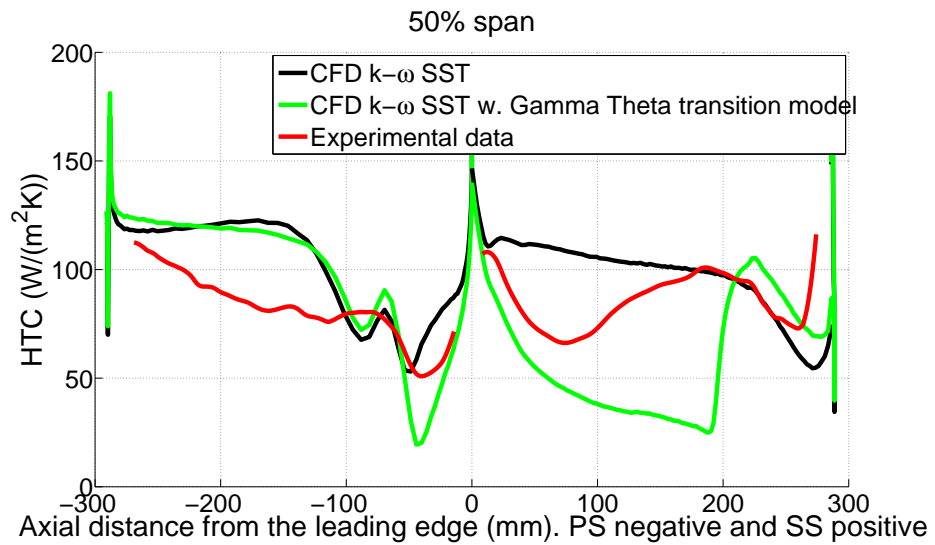

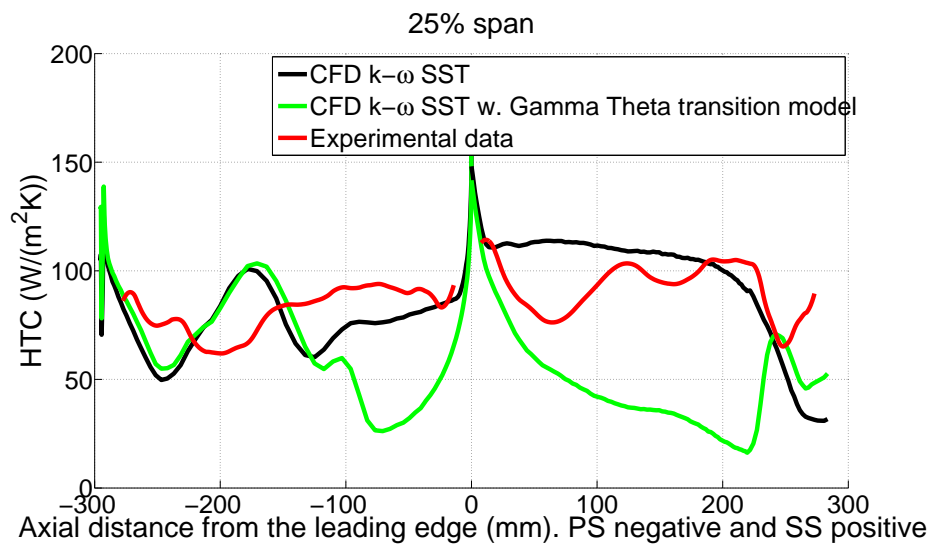

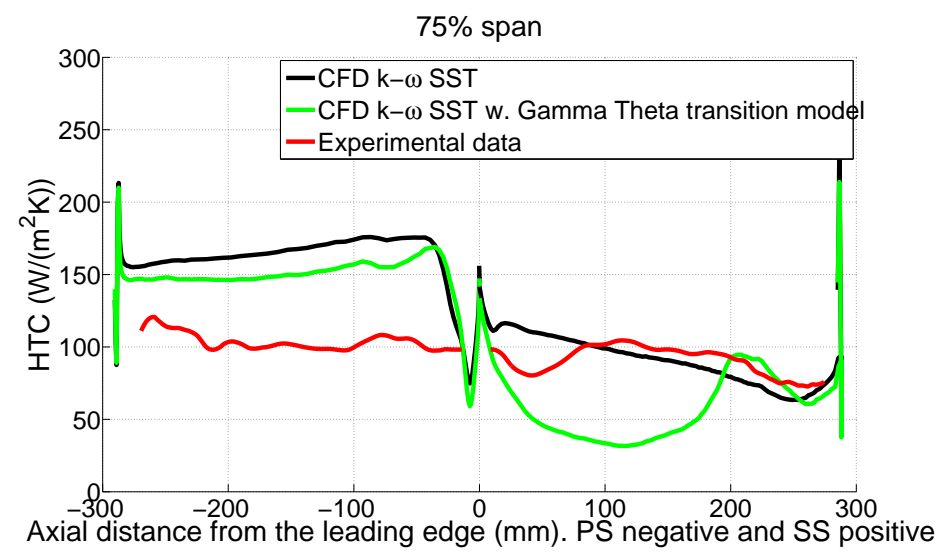

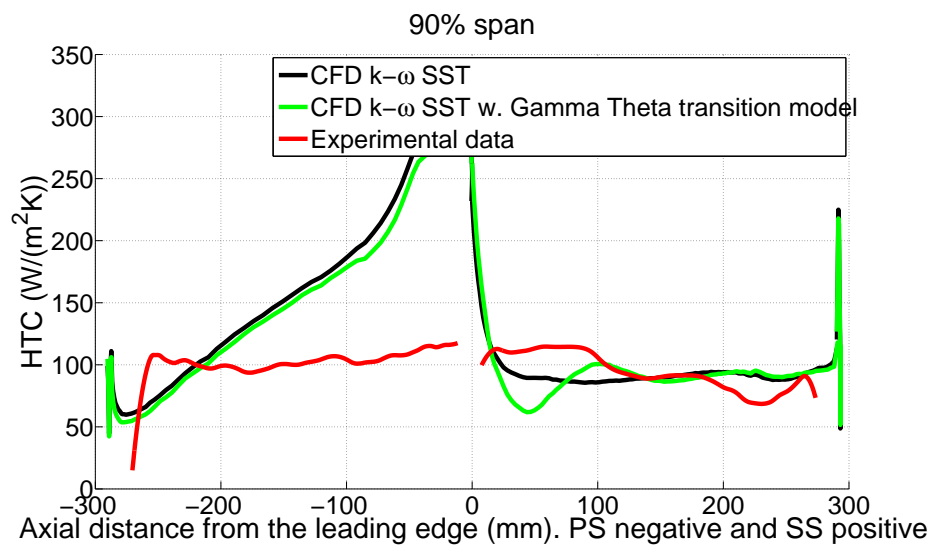

Figure 10. CFD and experimental data for $10 \%, 25 \%, 50 \%, 75 \%$ and $90 \%$ radial span. 


\section{Conclusion}

Experiments have been conducted, studying the heat transfer of the vane situated in an ITD. The results have been compared to two numerical analysis and are presented in this paper. The comparison demonstrates the challenges embedded in predicting a flow with complex structures interacting with each other and the main flow. The suction side heat transfer distribution is predicted relatively well with a few discrepancies while the pressure side is more difficult to predict.

It is found that the influence of the thermal radiation in the calculation of the convection heat transfer coefficient is important. Neglecting the radiation leads to an additional error in the determination of the heat transfer coefficient with about $5 \% 10 \%$ in current case.

\section{References}

${ }^{1}$ C. Arroyo Osso, (2009) "Aerothermal Inestigation of an Intermediate Duct", PhD Thesis, Chalmers University of Technology, Gothenburg, Sweden

${ }^{2}$ M. Johansson, V. Chernoray, L. Ström, J. Larsson, H. Abrahamsson "Experimental and Numerical Investigation of an Aerodynamically Loaded Guide Vane in a Turbine Duct", Proc. of ASME TURBO EXPO 2011, Paper no GT2011-46221

${ }^{3}$ L. Axelsson, (2009) "Experimental Investigation of the Flow Field in an Aggressive Intermediate Duct", PhD Thesis, Chalmers University of Technology, Gothenburg, Sweden 